\title{
Kommentar
}

\section{Selbstorganisation contra Studienreform}

Nicht zufällig sind die »Mainzer Beschlüsse « des Fakultätentages der juristischen Fakultären der BRD und Westberlins vom Februar 69 in dem Semester verabschiedet worden, in dem die Hochschulrevolte allenthalben endlich auch die juristischen Fakultäten erreichte. Auch in diesem Sinne waren die Beschlüsse * Reaktion *. Zu diesem Zeitpunkt allerdings wurde Inhalt und Ausbildungsform des juristischen Studiums so radikal in Frage gestellt, daß eine Besänftigung mittels einer die wahre Misere verschleiernden, auf totale Anpassung abzielenden technokratischen »Studienreform*, wie sie in München und Mainz von aufgeschredkten Ordinarien konzipiert wurde, nicht mehr verfing.

Nicht zufällig auch erwachten die juristischen Fakultäten dermaßen spät aus dem liebgewordenen Trott des Ausbildungsganges, der - nicht wesentlich anders als 50 Jahre zuvor - durch professoral verordneten *Rechrskundeunterricht * und beim Repetitor eingepaukter Subsumptionstechnik charakterisiert werden kann. Denn diese Fakultäten haben sich überall und immer als Hochburgen der Reaktion bewährt, was wesentlich durch die primäre und sekundäre Sozialisation der Jurastudenten und Juristen begründbar ist.

Die Einsicht, auch und gerade an den juristischen Fakultäten grundlegende inhaltliche wie instirutionelle Veränderungen herbeiführen zu müssen, wurde nicht erst im Wintersemester 68/69 geboren. Aber erst in diesem Semester wurde klar, daß neue Methoden gefunden werden mußten, da die bisherigen Ansätze gescheitert waren:

Der Versuch etwa, die »Kritische Universitär «, die nach dem heißen Juni 1967 in Berlin gegründet wurde, auch an der dortigen juristischen Fakultät zu etablieren, mußte zwangsläufig fehlschlagen. Verkürzt gesagt, hatte dieser Versuch darin bestanden, daß eine elitäre Gruppe von kritisch gewordenen Individuen losgelöst von den realen Ausbildungsbedingungen und den Bedürfnissen der Jura-Studenten seminarmarxistische Arbeitszirkel gründete, in denen abstrakte Ideologiekritik unter Zuhilfenahme von linksliberalen Autoritäten betrieben wurde. Dadurch wurden keine Veränderungen, sondern bestenfalls eine Kompensation der Unlust jener Individuen bewirkt.

Hierzu gesellte sich die Erfahrung mit der »Mauschelpolitik«, wie sie in Form von Studienreformkommissionen, Anträgen auf Fakultätssitzungen und Sprechstundengesprächen mit einzelnen Professoren geübt wurde: die im Herbst 67 an der juristischen Fakultät der FU gegründete Studienreformkommission war zur Diskussion (geschweige denn zur Realisierung) längerfristiger Reformen schon deshalb nicht imstande, weil sie von professoraler Seite monatelang boykottiert wurde. Bis zu Beginn des Wintersemesters $68 / 69$ beschäftigte die Kommission sich etwa mit Fragen, ob die Vorlesung Römische Rechrsgeschichte im 2. oder im 4. Semester gelesen werden sollte. Groß propagierte $»$ Reformen «, wie etwa die 
Institutionalisierung von Repetitorien und Klausurenkursen im Ausbildungsprogramm dienten ausschließlich dazu, den Studiengang reibungsloser zu gestalten und ihn an die Erfordernisse des Staarsexamens anzugleichen. Anträge des (in Berlin einzigen) studentischen Vertreters in Fakultätssitzungen wurden regelmäßig bei einer Gegenstimme (die des Antragstellers) abgelehnt. Prof. Betrermann scheute sich nicht, solche Anträge (etwa auf Offentlichkeit von Fakultätssitzungen) als den Versuch der vorsätzlichen Behinderung der Fakultätsarbeit zu qualifizieren.

Diese Erfahrungen produzierten die Einsicht, daß Veränderungen nur dann möglich sein würden, wenn eine große Anzahl von Jurastudenten ihrer Unterdrükkung und damit ihren Unterdrückern den Kampf ansagen würden, d.h. die -Gemeinschaft von Lehrenden und Lernenden * aufkündigen und als Gemeinschaft von Herren und Knechten entlarven würden.

Eine darauf gerichtete Strategie mußte zum einen die inhaltliche und lernorganisatorische Misere der Ausbildung in den Lehrveranstaltungen selbst aufzeigen, zum andern den berechtigten Forderungen nach institutionellen Veränderungen durch Vollversammlungen und reach-ins eine Massenbasis geben. Eine Praxis, der eine kritische Theorie zugrundeliegt, die die Krise von Staat, Gesellschaft und Recht zu analysieren versucht und in der Einlösung eines geschichtlich emanzipatorischen Anspruchs die Alternative zu einer autoritären Gesellschaft sieht, kann nicht affirmativ sein. Sie wird auch einen Handlungsrahmen nicht akzeptieren, der seine Rechts- und Anstandsregeln nicht legitimieren kann. So müssen die Akrionen durch einen wie auch immer gearteten Regelverstoß gekennzeichnet sein, dessen Vermittlung allerdings bei Studenten, deren Studium in $*$ Regelkunde« besteht, größere Schwierigkeiten macht als bei allen anderen Studenten.

In Berlin boten sich die im Wintersemester $68 / 69$ angeserzten Lehrveranstaltungen für Rechıssoziologie exemplarisch als Einstieg an, um die Kritik zu formulieren. Die vorgesehene Trennung in Vorlesung (zur "Stoffvermitrlung*) und Seminar (zur *Diskussion *) wurde durch einen Beschluß der Teilnehmer abgelehnt, der die elitäre Struktur des Seminars in gleicher Weise ablehnte wie die autoritäre der Vorlesung. Der Dozent sah sich genörigt, einer Verfahrensordnung zuzustimmen, die die inhaltliche Bestimmung der Arbeitsthemen des neugeschaffenen Großcolloquiums durch die Teilnehmer, eine studentische Diskussionsleitung und die kollektive Leistungsbewertung für den Scheinerwerb vorsah. Ein stetig anwachsender Kreis von Studenten bereitere die einzeinen Sitzungen inhalelich vor und erfüllte dadurch nicht die Absichr des Dozenten, daß die Studenten sich tor laufen und aus Informationsmangel schließlich reumütig wieder nach der Autorität rufen würden. Durch die Arbeit an und in diesem Seminar machten die Studenten die für die Folgezeit bedeutsame Erfahrung, wie eine relativ repressionsfreie Kollektivarbeit gleichzeitig bewußtseinsfördernd und mobilisierend nach außen wirken kann. Es bildete sich die *ad-hoc-Gruppe an der juristischen Fakultät der FU «, die inzwischen zu einem Trauma für Ordinarien und Administration und zu einer avant-garde der studentischen Reformarbeit geworden ist.

Die studentischen Mitglieder der Berliner Studienreformkommission regten in dem Rahmenplan zur Reform der juristischen Ausbildung « die Diskussion um längerfristige Perspektiven der Reformarbeit an. Dieses Konzept sieht eine Einheitsausbildung in vier Phasen vor: dreisemestriges sozialwissenschaftliches Propädeutikum, Einübung in die Rechtsmaterie auf breiter Basis unter größtmöglicher Aufhebung der Trennung der Rechtsgebiete und mit ideologiekritischem Vorzeichen, zweisemestriges Praktikum bei ständiger Theoriebezogenheit 
der praktischen Erfahrungen und schließlich eine Spezialisierungs- und Vertiefungsphase mit Wahlmöglichkeit für *Justiz «, *Wirtschaft « oder *Verwaltung Examina sollten nach diesem Konzept zugunsten einer begleitenden Studienkontrolle mit kollektiver Leistungsbewertung abgebaut werden. Arbeitsgemeinschaften sollten die zentralen Lehrveranstaltungen sein, Vorlesungen nur in der Form von Einführungs - und Vertiefungsvorlesungen in Zusammenhang mit Arbeitsgruppen beibehalten werden.

Uber die Unmöglichkeit, ein solches Konzept kurzfristig durchsetzen zu können, war man sich klar. Die Initiativen mußten von Studenten und Assistenten allein ausgehen. Der Forderung nach einem autonomen studentischen Sektor innerhalb der Ausbildung wurde die Legitimationsgrundlage gegeben.

Die Professoren der Studienreformkommission verweigerten die verbindliche Diskussion über den Rahmenplan. Startdessen verbor die Fakultät die weitere Arbeit des Rechtssoziologie-Colloquiums in dem Augenblidk, wo die Teilnehmer einen Praxisbezug ihrer theoretischen Arbeit herstellten und über die Repressionsfunktion der $" Z$ wangsezmatrikulation « diskutierten, die seit 1966 an der Berliner Fakultät praktiziert wurde, obwohl das Verwaltungsgericht Berlin in einer bis heute nicht rechtskräftigen Entscheidung eine befristete Zulassung für rechtswidrig erklärt hatte.

Der Forderung, über diese allein die Studenten betreffenden Frage auf einer öffentlichen Fakultätsitzung zu beraten, wurde abgelehnt. Daraufhin beschloß die studentische Vollversammlung Anfang Dezember, die Ơfentlichkeit der Sitzung gewaltsam herzustellen.

Dies gab den Ausschlag, die ersten Ordnungsverfahren nach dem Berliner *Vorschaltgesetz " vom Okt. 68 gegen gewählte studentische Vertreter der Juristischen Fakultät einzuleiten, mit der Begründung, sie hätten durch Äußerungen im *Juristen-Blatt ", dem Organ der ad-hoc-Gruppe, den Lehr- und Forschungsbetrieb erheblich gestört.

Die Ordinarienherrschaft mußte so reagieren, wie es zu erwarten war: der Tatsache, daß Studenten ihre Lernfreiheit praktiziert und die Konflikte offenbar gemacht hatten, konnte sie nur den administrativen $Z$ wang entgegensetzen. Als schließlich im Januar die ersten Relegationen aus politischen Gründen seit Bestehen der Bundesrepublik ausgesprochen wurden, traten die Jura-Studenten in großer $Z$ ahl aus ihrer traditionellen politischen Lethargie heraus und verkündeten den aktiven Streik bis zur Rüdknahme der Relegationen. Die Fakultät reagierte mit Aussperrung. In dem so geschaffenen Freiraum konnten eigenverantwortliche studentische Arbeitskreise gegründet werden, die mit der Kritik an den einzelnen Rechtsdisziplinen begannen. Um die renitent gewordene Studentenschaft zu befrieden, verkündere die Fakultär zu Ende des Semesters, daß im Sommersemester ein vexperimentelles Reformsemester " stattinden solle. Das von den Vertretern der einzelnen Fachrichtungen vorgetragene Reformprogramm entsprach nicht einmal den Forderungen, die die Studenten vor drei Jahren gestellt hatten: einige Vorlesungen sollten in Form von Colloquien abgehalten werden, ein methodologisches Seminar sollte veranstaltet werden, zwei Klausuren (statt einer Klausur und einer Hausarbeit) sollten zum Scheinerwerb ausreichend sein - die studentischen Forderungen nach wissenschaftstheoretischer Neubestimmung der Rechtswissenschaft, nach Praxisbezug der Arbeit, nach Autoritätsabbau in den Lehrveranstaltungen, nach neuen Lehr- und Lernmethoden wurden nicht verstanden.

Als weiteres Beispiel für das Aufbrechen der Konflikte an den juristischen Fakultäten mögen die Erfahrungen an der Frankfurter Fakultär im gleichen Seme- 
ster dienen. Auch hier ging die Strategie der mehrheirlich links besetzten Fachschaft (die in Frankfurt wie auch an anderen Fakultäten gleichzeitig die Funktion einer ad-hoc-Gruppe einnimmt) dahin, durch die Konfrontation mit einer studentischen Minderheit den bestehenden Lehr- und Ausbildungsbetrieb einer Kritik zu unterziehen, um zum Bewußtsein seiner selbst zu gelangen. Auch hier wurde im Wintersemester erkannt, daß nur eine Selbstorganisation der Studierenden, die die langfristig konzipierten Reformvorstellungen vorwegnimms, eine restriktive Studienreform sinnvoll bekämpfen und überwinden kann.

Die hoffnungslose Arbeitsplatzsituation an der Frankfurter Fakultät verbunden mit der Gefahr, daß das Juristische Seminar wegen des Umzugs in das neue Juridicum für ein halbes Jahr schließen würde, führte zu einem eintägigen Warnstreik, wobei den Studenten vermittelt werden konnte, daß nicht über parlamentarische Diskussionen und Resolutionen, sondern nur durch direkte Aktionen auf die Mißstände aufmeriksam gemacht werden kann.

Der konservativ-beschränkte, von den Interessen der Studenten abstrahierende Rechtskunde-Unterricht wurde exemplarisch in einer Vorlesung *Einführung in die Rechtswissenschaft « dargestellt. Konsequentes Infragestellen der professoralen Ausführungen und Widerstand gegen die autoritäre Vorlesungs-Form führten schließlich zur Niederlegung der Lehrveranstaltung. Der willkürliche Charakter des Prüfungswesens konnte durch die Konfrontation mit einem Professor, der erwiesenermaßen seine Seminarteilnehmer in der mündlichen Prüfung bevorzugt und ausschließlich aus seinen Interessengebieten prüft, in dessen Vorlesung dargestellt werden.

Die durch diese interessenbezogenen Aufklärungsaktionen mobilisierten Studenten, im wesentlichen Anfangssemester, organisierten sich in Arbeitskreisen, die inhaltlich an die aufgezeigten Problemstellungen anknüptten und zu verwirklichen versuchten, was bislang nur versucht wurde: kollektive Lernprozesse, herrschaftsf reies Lernen, Verknüpfung von Theorie und Praxis, interdisziplinäre Zusammenarbeit.

An fast allen juristischen Fakultäten der Bundesrepublik sind folgende Tendenzen durchgängig zu beobachten: abstrakte Reformdiskussionen sind als sinnlos, verzögernd und verschleiernd erkannt und werden, wenn überhaupt, nur noch als Legitimation gegenüber Studenten praktiziert, die die frustierenden Erfahrungen der Kommissionsdiskussionen noch nicht erkannt haben: daß man es wauch auf diesem Wege versucht habe «.

Stattdessen haben sich fast überall Basisgruppen gebildet, die mit oder gegen die traditionellen Fachschaften studentische Selbstorganisationen betreiben, einzelne relevante Lehrveranstaltungen von innen heraus umfunktionieren und den Lernbedürfnissen der Studenten, nicht aber einem vorgegebenen Examensritus anpassen. Daneben arbeiten studentische Arbeitsgruppen, womöglich unter Verwertung des Informationsvorsprungs von Assistenten und Referendaren. In den meisten Fällen arbeiten die Basisgruppen der Juristen zusammen mit Ermittlungsausschüssen, Rechtshilfekomitees und Strafvollzugs-Arbeitskreisen auch für die Justizkampagne und versuchen eine wechselseitige Vermittlung des universitären und außeruniversitären Bereichs herzustellen. So wird z. B. in Frankfurt eine unmittelbare, auch personelle, Verbindung $z$ wischen der praktisch tätigen Arbeitsgruppe Gefangenenbetreuung, dem Arbeitskreis Kriminologie und dem kritischen Teil der Teilnehmer von Strafrechtsvorlesungen- und -übungen angestrebt.

Aber auch die Schwierigkeiten sind an den einzelnen juristischen Fakultäten relativ homogen: einer selbsttätigen Praxis bewußt gewordener Studenten steht eine 
schizoide Sirutation entgegen: die internen Integrationsmechanismen (Examen, Ubungen zum Scheinerwerb), das externe Anpassungssystem (juristische Berufe) sowie die ständige Gefahr des administrativen oder judikativen Eingreifens (Ordnungs- und Strafverfahren, Stipendienentzug) führen zu einer für den einzelnen untragbaren psychischen und physischen Belastung. $\mathrm{Da}$ die Arbeitskreise bisher nirgends mit Gratifikationen in Form von Scheinen bedacht werden und parallel zum Ausbildungsweg laufen, erfordert die Teilnahme Doppelarbeit. Hinzu kommt, daß die traditionellen juristischen Berufe kaum Möglichkeiten bieten, um radikal verändernd auf die Gesellschaft einzuwirken, wie es sich aus einer kritischen Ausbildung zwangsläufig ergeben müßte.

Die Durchführung des Streiks in Berlin machte offenbar, wie sehr diese Schwierigkeiten und Widerstände die politische Arbeir behindern.

Um dem zu begegnen, mußte aus der ad-hoc-Organisation eine verbindliche, langfristige Organisation geschaffen werden: aus der Einsicht, daß der Druck durch Kollektive besser würde aufgefangen werden können und ein Nebeneinander von "politischer " und "privater * Existenz nicht möglich ist, bildeten sich Wohngemeinschaften und Lernkommunen. Ein zentrales Organisations-Komitee sollte die verschiedenen Aktivitäten der Gruppe koordinieren und für besseren Informationsfluß sorgen. $\mathrm{Zu}$ Beginn des Sommersemesters bestanden diese Aktivitäten u. a. in folgendem: mehrere Arbeitskreise zur Politischen Okonomie; Arbeitskreise, die sich inhaltlich an einzelne Lehrveranstaltungen orientierten, um auf diese Veranstaltungen einzuwirken; Redaktion des "Juristen-Blatt ; Beratung von Anfangssemestern; Arbeitskreis $\gg$ Arbeitsrecht«, um eine spätere $\mathrm{Zu-}$ sammenarbeit mit außeruniversitären Basisgruppen vorzubereiten usf. Wie in allen studentischen Arbeitsgruppen lagen die internen Schwierigkeiten besonders darin, keine neuen Autoritäten, "Ersatzordinarien «, aufkommen zu lassen, die psychischen Hemmungen insbesondere jüngerer Semester zu berücksichtigen und sich durch den im Semester ständig herrschenden Aktionsdruds nicht von der substantiellen theoretischen Arbeit abbringen zu lassen.

Für die Arbeit der (zahlenmäßig ständig anwachsenden) Basisgruppen der Juristen ergeben sich folgende Perspektiven:

x. Die inhaltliche wie methodische Kritik in Lehrveranstaltungen und tendenziell ihre Umfunktionierung muß fortgesetzt werden, sie ist ein wesentlicher Mobilisierungsfaktor, insbesondere bei Veranstaltungen für Anfangssemester.

2. Studentische Arbeitskreise unter Zuhilfenahme von Referendaren und Assistenten müssen einzelne Rechtsdisziplinen ideologiekritisch untersuchen und dabei auf praktische Projekte hinarbeiten.

3. Um das "Scheine-System zu unterlaufen, sollten Musterlösungen von Hausarbeiten rechtzeitig vor dem Abgabetermin veröffentlicht werden. Klausuren sollten nicht mehr nur *juristisch «, sondern »politisch " gelöst werden. Es sollte (wie etwa bei den Soziologen in Frankfurt) der Versuch gemacht werden, Klausuren kollektiv zu schreiben.

4. Um das externe Integrationssystem der juristischen Berufe tendenziell aufzulösen, wäre zu prüfen, ob die historisch diffamierte Taktik des Kampfes in des Apparaten eine realistische Möglichkeit ist (unter Zusammenarbeit mit den Basisgruppen der Referendare); es wäre zu prüfen, wie Justizopfer politisiert werden können (unter Zusammenarbeit mit den Strafrollzugsgruppen) und ob Aufklärung in der Produktionssphäre (Arbeiterberatung) für die Mobilisierung relevant sein kann, wobei die unterdrüdkende Funktion des Arbeitsrechts über konkrete Konflikte zu vermitteln wäre.

5. Die juristischen Basisgruppen sollten sich ihrer Rolle als avantgarde-Kader 
bewußt werden und daraus verbindliche Organisationsformen ableiten, die nicht zuletzt auch den Existenzschutz des einzelnen berücksichtigen müßten. Die Basisgruppen der einzelnen Fakultäten sollten durch eine überregionale Organisation eine bessere Kommunikation herstellen. Wieweit dies mit Hilfe des vds geleistet werden kann, müßte geprü̈t werden.

Christopher Heinz Michael Gesell

Heinrich Schacht

\section{Ein go-in im Apparat}

Immer noch gilt der Justizapparat als geschlossener Wall gegen jeden Ansarz von Emanzipation und Demokratie. In Berlin aber ist durch eine kollektive Aktion zum ersten Mal ein Durchbruch gelungen. Während sich alle "Welt« über das Rehse-Urteil durch das Oske-Gericht erregte, um kurz darauf wieder in den gewohnten "Trott« zu verfallen, versuchte eine Gruppe von Referendaren - zunächst unter Einhaltung der üblichen Spielregeln - eine öffentliche Diskussion über den Freispruch und die dahinterstehende Ideologie herbeizuführen. In Flugblättern - an alle Referendare und die Justizverwaltung verteilt - wurde zur Diskussion aufgerufen. Als niemand reagierte, folgte eine Petition an den Kammergerichtspräsidenten, die von über I 20 Referendaren unterschrieben war. Dieser Vorstoß kam offensichtlich für die Bosse der Justizverwaltung und für Oske völlig unerwartet. Denn die geforderte Diskussion wurde zugesagt, dann abgelehnt, dann zugesagt und wieder abgelehnt. Man glaubte wohl, daß die Referendare von alleine wieder Ruhe geben würden.

Die Referendare hatten inzwischen längst den exemplarischen Wert des RehseUrteils erkannt: in ihm wird die Unfähigkeit der Juristen zur bewußten Konfliktlösung deutlich; in ihm zeigen sich die verheerenden Folgen des stumpfsinnigen Gesetzespositivismus, unkritischer Autoritätsgläubigkeit und reaktionärer Vorurteile der Justizbeamten; in ihm offenbart sich ungeschminkt die Diskrepanz zwischen liberaler Rechtsstaatsideologie und konservativ-autoritärer Praxis.

Deswegen konstituierten sich Berliner Referendare zu einer ad-hoc Gruppe und planten ein go-in in eine Arbeitsgemeinschaft für Referendare, in der immer noch der Justiznachwuchs von dem Rehse-Richter Oske ausgebilder wird.

Bei der eingehenden Vorbereitung in der Gruppe ergaben sich Meinungsverschiedenheiten über die Methode der im Prinzip als notwendig erkannten Aktion. Zum einen sprach die Gefahr von Disziplinarmaßnahmen dafür, jedwede Spiclregelverletzung auszuschließen - also keine Gewalt gegen Justizbeamte anzuwenden und nichr das Risiko eines Hausfriedensbruches einzugehen. Zum anderen wurde die Notwendigkeit der Regelverletzung betont, da nur auf diese Weise ein Druck auf den Apparat ausgeübt werden kann. Nur so muß er sich zwischen der Verfolgung von Gesetzesverstößen und der Forderung nach Diskussion und Herstellung von Offentlichkeit entscheiden. Diese Meinung setzte sich schließlich durch, und das go-in fand statr.

Funk, Fernsehen und Presse berichteten über das go-in; eine kleine Anfrage der 